\title{
Quantum electromechanics: Quantum tunneling near resonance and qubits from buckling nanobars
}

\author{
Sergey Savel'ev ${ }^{(1,2)}$, Xuedong $\mathrm{Hu}^{(1,3)}$, A. Kasumov ${ }^{(4)}$, and Franco Nori ${ }^{(1,5)}$ \\ (1) Frontier Research System, The Institute of Physical and Chemical Research (RIKEN), Wako-shi, Saitama, 351-0198, Japan \\ ${ }^{(2)}$ Department of Physics, Loughborough University, Loughborough LE11 3TU, UK \\ (3) Department of Physics, University at Buffalo, SUNY, Buffalo, NY 14260-1500, USA \\ (4) Laboratoire de Physique des Solides, Associé au CNRS, Université Paris-Sud, F-91405, Orsay, France \\ (4) Center for Theoretical Physics, Department of Physics, \\ University of Michigan, Ann Arbor, MI 48109-1040, USA
}

(Dated: November 12, 2018)

\begin{abstract}
Analyzing recent experimental results ${ }^{1.2}$, we find similar behaviors and a deep analogy between three-junction superconducting qubits and suspended carbon nanotubes. When these different systems are ac-driven near their resonances, the resonance single-peak, observed at weak driving, splits into two sub-peaks (Fig. 1) when the driving increases. This unusual behavior can be explained by considering quantum tunneling in a double well potential for both systems. Inspired by these experiments, we propose a mechanical qubit based on buckling nanobars - a NEMS so small as to be quantum coherent. To establish buckling nanobars as legitimate candidates for qubits, we calculate the effective buckling potential that produces the two-level system and identify the tunnel coupling between the two local states. We propose different designs of nanomechanical qubits and describe how they can be manipulated. Also, we outline possible decoherence channels and detection schemes. A comparison between nanobars and well studied superconducting qubits suggests several future experiments on quantum electromechanics.
\end{abstract}

PACS numbers: $85.85 .+\mathrm{j}$

\section{INTRODUCTION}

Micro- and nano-electromechanical systems (MEMS and NEMS) have attracted widespread attention because of their broad spectrum of functionalities, their tiny submicron sizes, and their unique position bridging microelectronic and mechanical functions ${ }^{3}$. Sophisticated tools ranging from mirrors and sensors, to motors and multifunctional devices have been fabricated ${ }^{4.5}$. As the size of these devices shrinks, experimental studies of NEMs are rapidly approaching the quantum limit of mechanical oscillations ${ }^{5.6 .7 .8 .9}$, where quantum coherence and superposition should result in quantum parallelism and the possibility of information processing.

The emergence of quantum electromechanical devices (see, e.g., Ref 3.4 .6 .7 .8 .9 and references therein) brings both challenges, such as the inevitable and ubiquitous quantum noise, and promises, such as macroscopic quantum coherence ${ }^{8.10,11}$ or quantum teleportation ${ }^{12.13}$. Indeed, during the past several years the quantum mechanical properties of NEMS and how they can be coupled to other quantum mechanical objects have been very actively studied $14,15,16,17.18 .19$. The experimental pursuit of these studies has so far focused on cooling a device to reach states where quantum fluctuations in the lowest energy eigenmode dominate over thermal fluctuations ${ }^{6.15}$. Such eigenmodes are generally harmonic-oscillator modes with equal energy-spacings and follow bosonic statistics.

Among NEMS there also exist systems that can be well approximated by two levels in the quantum limit, so that they might be candidates for qubits. For example, when a longitudinal strain above a certain critical value is ap- plied to a small bar with one or two ends fixed, there exist two degenerate buckling modes, as schematically shown in Fig. 2. In the quantum mechanical limit, these two modes represent the two lowest-energy states. They can be well separated from the higher-energy excited states, so that at low temperatures the buckling nanobar can be properly described as a two-level system. Furthermore, since nanobars can be charged or can carry electrical current, the electric or magnetic field can be conveniently used to manipulate their quantum states.

The fascinating prospect of observing quantum coherent phenomena in a macroscopic mechanical oscillator is a main motivation of this study. To achieve this goal, a NEMS needs to possess at least two general attributes: small size and a high fundamental frequency. A carbon nanotube is a natural candidate: it is very thin (and can be very short) while still being stiff 20 (thus having high vibration frequency) because of the strong covalent bonds between carbon atoms within the graphene sheet 21.22 . However, without strong experimental support, it might be hard to judge the feasibility of achieving macroscopic quantum coherence in NEMS. In contrast to NEMS, experimental evidence has demonstrated that superconducting charge ${ }^{23}$, flux $^{24}$, and phase ${ }^{25}$ qubits, using Superconducting Quantum Interference Device (SQUID), do exhibit quantum coherence. Thus, it is important to look for possible similarities in the behavior of superconducting qubits and NEMS. This should help future prospects to observe quantum coherence in NEMS. 


\section{SPLITTING RESONANCE AS A MANIFESTATION OF QUANTUM TUNNELLING IN THREE-JUNCTION SQUIDS AND NEMS}

Experiments ${ }^{1}$ on suspended single-wall carbon nanotubes (with diameter of about $20 \mathrm{~nm}$ and length of about $1.7 \mu \mathrm{m}$ ) excited by an electromagnetic wave show a resonance peak with an unusual shape (Fig. 1a), for one of the fundamental harmonics $\omega_{0}$. As expected, a Lorentz-form resonance peak, that grows with increasing intensity of the ac field, was observed at weak drivings. Surprisingly, when further increasing the amplitude of the externally applied electromagnetic wave, this peak splits into two sub-peaks 1 . When further increasing the ac drive, these two sub-peaks gradually move away from each other, while their heights stop growing. It is important to stress that this phenomenon was observed at a frequency $\left(\omega_{0} / 2 \pi \sim 2 \mathrm{GHz}\right)$ and temperature $(T \sim 100 \mathrm{mK})$ where quantum effects start to dominate over thermal noise $\left(T \lesssim \hbar \omega_{0} / k_{B} \approx 100 \mathrm{mK}\right.$, with Boltzmann constant $k_{B}$ ). Also the dissipation in the system was quite low (quality factor $Q \sim 1500$ ), which is important to observe quantum effects. Note that the fundamental frequency of this device can be easily increased (at least by an order of magnitude, well into the operating frequency $1-15 \mathrm{GHz}$ of many superconducting qubits), for experimentally available carbon nanotubes with shorter length.

Interestingly, a similar phenomenon has been recently found $^{2}$ for an Al three-junction SQUID qubit coupled to a $\mathrm{Nb}$ resonant tank circuit (Fig. 1b). It was experimentally proven ${ }^{2}$, via the observation of quantum hysteresis (Landau-Zener transitions), that this circuit was operated in the quantum regime (at $\omega_{0} / 2 \pi \sim 20 \mathrm{MHz}$ and $T \gtrsim 10 \mathrm{mK}$ ), though for a worse ratio of quantum to thermal noise compared to the carbon nanotubes 1 (i.e., $\omega_{0} / T \sim$ one order of magnitude higher for the nanotube). When the magnetic flux in the SQUID was driven as $\Phi=\Phi_{\mathrm{dc}}+\Phi_{\mathrm{ac}} \cos (\omega t)$, the resonance in the response, probed via the tank voltage as a function of the dc flux, was found ${ }^{2}$ to exhibit a transformation from a Lorentz-form single-peak to a double-peak shape (Fig. 1b) in striking similarity to suspended driven carbon nanotubes ${ }^{1}$. Sweeping the dc flux in the SQUID corresponds to changing the fundamental frequency as

$$
\omega_{0}=\omega_{0}\left(\Phi_{\mathrm{dc}}=0\right)+\Delta \omega_{0}\left(\Phi_{\mathrm{dc}}\right)
$$

with $\Delta \omega_{0} \propto\left(\Phi_{\mathrm{dc}}-\Phi_{0} / 2\right)$ and flux quantum $\Phi_{0}$. Thus, we find that the measured ${ }^{1.2}$ response, near resonance, of both systems (carbon nanotube and three-junction SQUID qubit) depends on the difference $\omega-\omega_{0}$. This will be useful to establish below that the measurements for driven carbon nanotubes $\frac{1}{}$ and three-junction SQUID qubit $^{2}$ essentially probe the same effect.
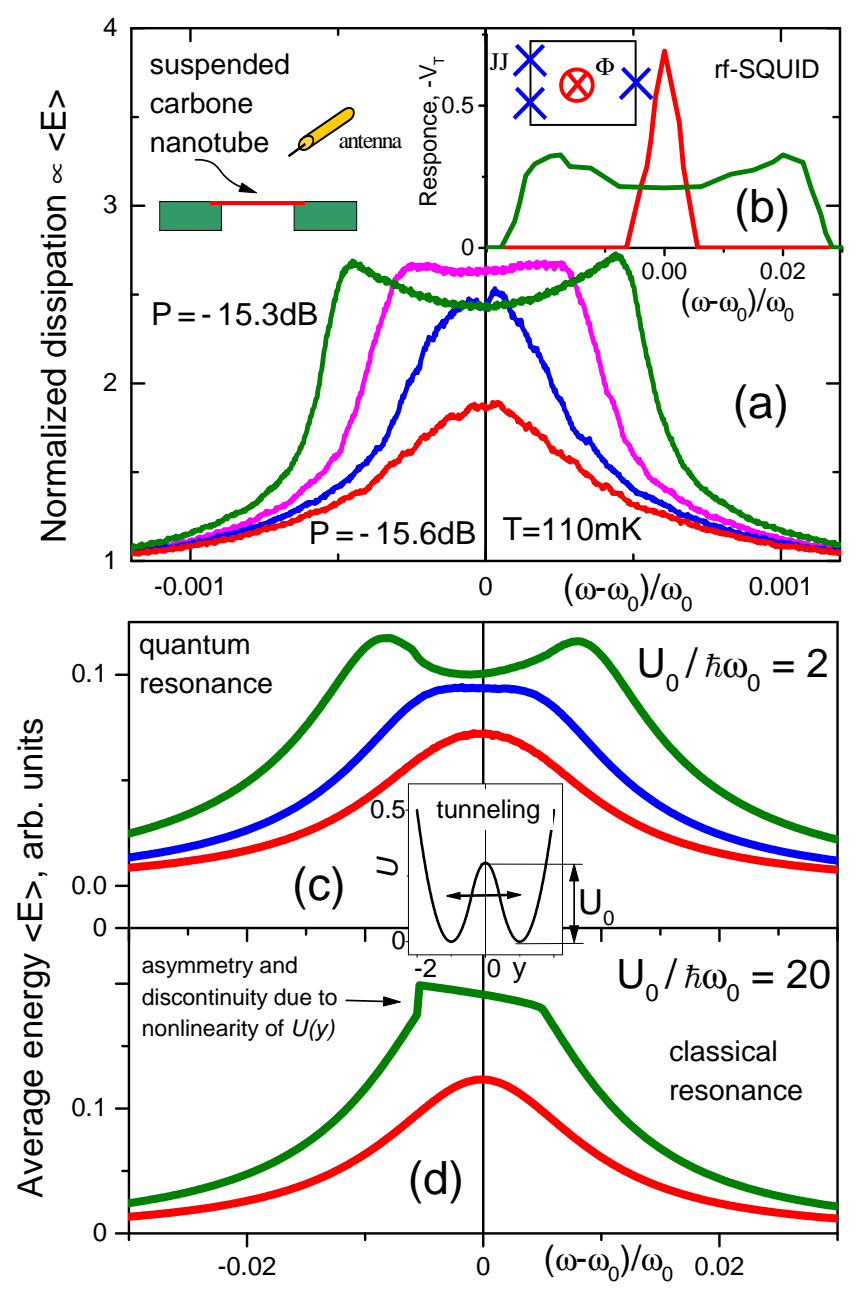

FIG. 1: (a) Experimental resonance $\frac{1}{1}$ of the effective dissipation (which is proportional to the average energy $\langle E\rangle$ ) as a function of the dimensionless frequency $\left(\omega-\omega_{0}\right) / \omega_{0}$, for a suspended carbon nanotube, for different intensities $P$ of the externally applied ac electromagnetic wave. (b): the resonance in the response of the three-junction SQUID probed by measuring ${ }^{2}$ the voltage for the resonant tank $V_{T}$ for different driving amplitudes as a function of $\left(\omega-\omega_{0}\right) / \omega_{0}$. For these different systems, a similar splitting of the resonance peak was observed ${ }^{1.2}$. Schematic diagrams for a driven suspended carbon nanotube and three junction SQUID are also shown at the top. The simulated resonance [average energy $\langle E\rangle$ versus the dimensionless frequency $\left.\left(\omega-\omega_{0}\right) / \omega_{0}\right]$ for quantum (c) and classical (d) particles moving in the double-well potential shown in the inset of (c). A quantum description agrees with experiments ${ }^{1.2}$ (Fig. 1a,b): For quantum particles, the standard Lorentz-form resonance peak for weak ac drives (c, red curve, $A=0.005$ ) splits into two sub-peaks due to tunnelling at stronger drives (blue curve, $A=0.01$; green curve, $A=0.0135)$. A classical description cannot describe experiments 1.2 (Fig. 1a,b): For classical particles, the weakdriving Lorentz-form peak (red curve, $A=0.005$ ) becomes asymmetric and exhibit discontinuous jumps $E(\omega)$ (green curve, $A=0.0135)$ due to the nonlinearity of $U(y)$. 
We propose how the incorporation of quantum tunnelling can be used to understand these still-unexplained experimental observations in nanotubes 1 and three junction SQUIDs ${ }^{2}$. In order to interpret the splitting of the resonance peak (Fig. 1a and 1b), we employ a quasiclassical model26 described by the equation of motion

$$
\ddot{y}+2 \lambda \dot{y}+\partial U(y) / \partial y=A \cos (\omega t),
$$

for the double-well potential shown in the inset in Fig. 1c, with small damping $\lambda=0.01$, and different driving amplitudes $A$. We assume that the particle (which mimics the buckling mode or current in the SQUID) can tunnel from one well to the other with transmission coefficient

$$
D=\exp \left[-\frac{2 \sqrt{2}}{\hbar} \int_{-a}^{a} \sqrt{U-E} d y\right],
$$

where $a$ is the classical turning point, and $E=(\dot{y})^{2} / 2+$ $U(y)$ is the energy of the particle. This minimal model allows to qualitatively describe the resonance of a quantum particle in a double well potential (a more complete, fully quantum mechanical, theory will be published elsewhere ${ }^{27}$ ) and also to obtain the transition to a classical description (Fig. 1c,d).

When the potential barrier $U_{0}$ is comparable with $\hbar \omega_{0}$, we find that the single resonance peak, at low driving, splits into two sub-peaks (Fig. 1c) for higher drives; in agreement with experimental findings 1.2 . The physical origin of this effect is the following: (i) At low driving the energy $E$ of the quantum particle (whether the SQUID or the nanotube) is also low and the probability of tunnelling is negligibly small, thus, the usual Lorentzform of the resonance occurs; (ii) When the driving (and, thus, energy) increases, the particle starts to tunnel between the wells. A tunnelling event is equivalent to an inversion around the origin, i.e., changing $y$ to $-y$ : like replacing the left well by the right one, or vice versa. As a result, right after a tunnelling event, the average power $\mathcal{P}=\langle\dot{y} F \cos \omega t\rangle$ the particle receives from the external force (e.g., the power absorption by the nanotube from the external electromagnetic wave) changes its sign $\mathcal{P} \rightarrow-\mathcal{P}$ and, hence, the energy, which increases before tunnelling, starts to decrease temporarily. Thus, the average energy $\langle E\rangle$ also decreases when the number of tunnelling events increases. This explains the minimum of $\langle E(\omega)\rangle$ at $\omega=\omega_{0}$ (instead of the maximum observed at weak driving) and also the splitting of the resonance peak - because the tunnelling frequency sharply increases at the resonance frequency $\omega_{0}$.

For the case of "more classical" particles, $U_{0} \gg \hbar \omega_{0}$, the probability of tunnelling is always very low. When driving increases, the particle (which is always located in the same well) begins to feel the strong nonlinearity of the potential $U(y)$, resulting in the energy dependence of the oscillation frequency $\omega_{0}(E)$. Instead of a split resonance, the resonance peak as a function of frequency shows the standard asymmetric shape with a sharp jump of $\langle E(\omega)\rangle$, associated with mechanical hysteresis 28 (Fig. 1d).
Therefore, we conclude that the two-peak resonance indicates that both the three-junction SQUID qubit ${ }^{2}$ and the suspended carbon nanotube $e^{\frac{1}{}}$, operate in the quantum regime. Thus, the technology for fabricating suspended buckled carbon nanotubes, working as nanomechanical qubits, already exists 1 . In view of the explosive growth of NEMS technology, below we discuss the prospect of such buckling charged nanobars (the clamping at the base ensures an anisotropic nanobar instead of an isotropic nanotube) as candidates of quantum bits for quantum information processing. Indeed, previous work on quantum buckling (e.g., 10.11 $)$ were not focused on operating these as qubits, which is our focus here ${ }^{29}$.

\section{CONTROLLING QUANTUM STATES OF A NANOROD IN A DOUBLE-WELL POTENTIAL.}

The double-well potential (Fig. 2a) corresponding to the buckling modes $y \sin \left[\pi l / l_{\max }\right]$ of a nanorod (Fig. $2 \mathrm{~b}$ ) takes the form:

$$
U(y)=\alpha y^{2}+\beta y^{4}+\frac{2 l_{\max } f_{\perp}}{\pi} y,
$$

where the parameters can be estimated ${ }^{30}$ as

$$
\begin{aligned}
\alpha(f) & =\frac{\pi^{2}}{4 l_{\max }}\left(f_{c}-f\right), \\
\beta(f) & =\frac{\pi^{4}}{64 l_{\max }^{3}}\left(4 f_{c}-3 f\right), \\
f_{c} & =\frac{I Y \pi^{2}}{l_{\max }^{2}} .
\end{aligned}
$$

Here, we introduce the tube length $l\left(0 \leq l \leq l_{\max }\right)$, the elastic modulus $Y$ and the moment of inertia $I$. To control the rod we need: (i) a longitudinal compressing force $f$ acting on the rod ends, and (ii) a transverse force $f_{\perp}$, which can be produced via, e.g., interacting the charged nanorod with an electric field.

For zero transverse force $f_{\perp}=0$, our qubit (Fig. 2b) is in its degeneracy point. Indeed, this potential has two minima, at $y= \pm y_{0}(f)= \pm \sqrt{\alpha / 2 \beta}$ that are separated by a potential barrier

$$
\Delta U(f)=\alpha^{2} / 4 \beta .
$$

The first two energy levels $E_{1}$ and $E_{2}$ in the right well can be estimated assuming a parabolic potential well shape $U \approx m \omega_{0}^{2}\left(y-y_{0}\right)^{2} / 2$ with

$$
\omega_{0}(f)=\left(\frac{U^{\prime \prime}\left(y_{0}\right)}{m}\right)^{1 / 2}=2\left(\frac{\alpha(f)}{m}\right)^{1 / 2}
$$

and the mass $m$ of the nanorod. Thus, we obtain

$$
E_{1}(f)=3 \hbar(\alpha(f) / m)^{1 / 2}
$$

and

$$
E_{2}(f)=5 E_{1}(f) / 3 .
$$


Due to quantum tunnelling between the left and right potential wells, $E_{1}$ splits into two levels

$$
E_{1}^{ \pm}=E_{1} \pm \hbar \Delta_{t}
$$

The tunnelling rate $\Delta_{t}$ between the left and right buckled states is

$$
\Delta_{t}(f) \approx \frac{2}{\pi} \sqrt{\frac{\alpha(f)}{m}} \exp \left(-\frac{\pi \sqrt{2 m}\left(\Delta U(f)-E_{1}(f)\right)}{2 \hbar \sqrt{\alpha(f)}}\right) .
$$

It is important to stress that the longitudinal force $f$ allows to control the tunnelling $\Delta_{t}(f)$ as well as the energy levels itself $E_{1}(f)$. The higher levels are well separated from the two lowest ones: $\left(E_{1}^{+}-E_{1}^{-}\right) /\left(E_{2}-E_{1}\right) \sim$ $\Delta_{t} / \hbar \omega_{0} \ll 1$. Changing the transverse force $f_{\perp}$ moves the system out of the degeneracy point, allowing to manipulate the proposed nano-mechanical qubit.

We now introduce another, more controllable, design of a nanorod qubit, which is also less affected by decoherence coming from the relative vibrations of the rod ends (Fig. 2c). In such a design we consider a "crossed" electric field having a component $E_{\|}$along the rod and another $E_{\perp}$ perpendicular to the rod, which is clamped to a substrate only by one end. Changing the charge of the top capacitor plate and the charge of the rod itself can control both the electric field, $E_{\|}$, on the rod and its gradient, $\partial E_{\|} / \partial x$. By adjusting the different knobs, we can tune the relative strength between thermal activation and quantum tunneling, allowing the observation of transition between these two regimes.

\section{MANIPULATION, DECOHERENCE, AND DETECTION OF MECHANICAL QUBITS}

The manipulation of the mechanical qubits can be achieved electrically (Fig. 3). For example, in analogy to the Cooper pair box ${ }^{23}$ (see table), one can prepare the nanobar qubit in the $|L\rangle$ state by setting a transverse electric field towards the right (assuming the nanobar is negatively charged). By suddenly turning off this electric field and bringing the system to the degeneracy point, the nanobar state is prepared in a coherent superposition of $(|R\rangle \pm|L\rangle) / \sqrt{2}$. Because of the tunnel splitting, the system then starts to oscillate coherently, with a frequency given by $\Delta_{t}$. Therefore, by detecting the nanobar position, as a function of $\Delta t$ (see Fig. 3), one can determine the coherent oscillation frequency and the system decoherence. Driven coherent transitions between the two qubit states can also be similarly achieved. A sinusoidal component can be added to the vertical electric field that is used to control the tunnel coupling between the $|L\rangle$ and $|R\rangle$ states, in analogy to the microwave driving force on the Josephson coupling in a Cooper pair box. The study of free and driven coherent oscillations of a nanobar would help clarify its quantum coherence properties and demonstrate its feasibility as a qubit.
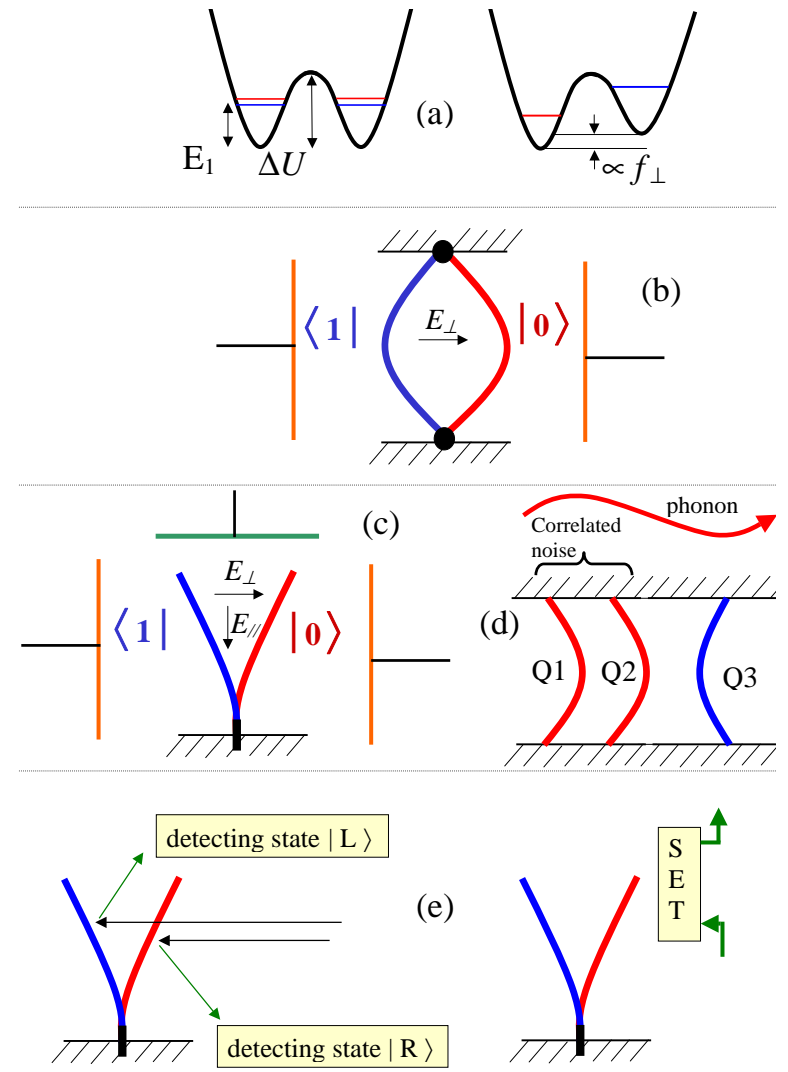

FIG. 2: (a) Double-well potential for a buckled nanobar. Due to tunnelling from the right potential well to the left one, the lowest energy level is split into two levels for $f_{\perp}=0$ as shown in the left panel. The lowest (blue) and the first excited (red) levels correspond to the symmetric and antisymmetric combinations of the wave functions localized in the left and right potential wells. The energy level splitting between the left and right states can be controlled by the transverse force $f_{\perp}$ as shown in the top right panel. (b) A buckled rod qubit where the compressed force applied to the rod ends controls the potential shape $[\alpha$ and $\beta$ in Eq. (5) $]$ and, therefore, the energy splitting at the degeneracy point. The transverse force $f_{\perp}$ allows to drive the bar to a degeneracy point. (c) Another proposed design for a buckled-rod qubit. In this case the possible decoherence originating from the relative vibration of the top and bottom rod holders can be avoided. By changing the charge of the top capacitor plate and the charge of the rod itself it is possible to independently control both the parallelto-rod electric field $E_{\|}$and its gradient. This design allows a high level of control of both the tunnelling and the energy splitting. (d) Correlated noise produced by some phonons can be eliminated using a decoherence-free subspace: qubits Q1 and Q2 can be associated with one logic qubit. (e) Singleshot measurements could be done via either single electron transistor (SET) or photon reflection.

Universal quantum computing requires two-qubit operations. For charged nanobars, the inter-qubit interaction comes naturally in terms of the electric dipole interaction between the bars, quite similar to the dipole interaction used in other proposed qubits. In the case 


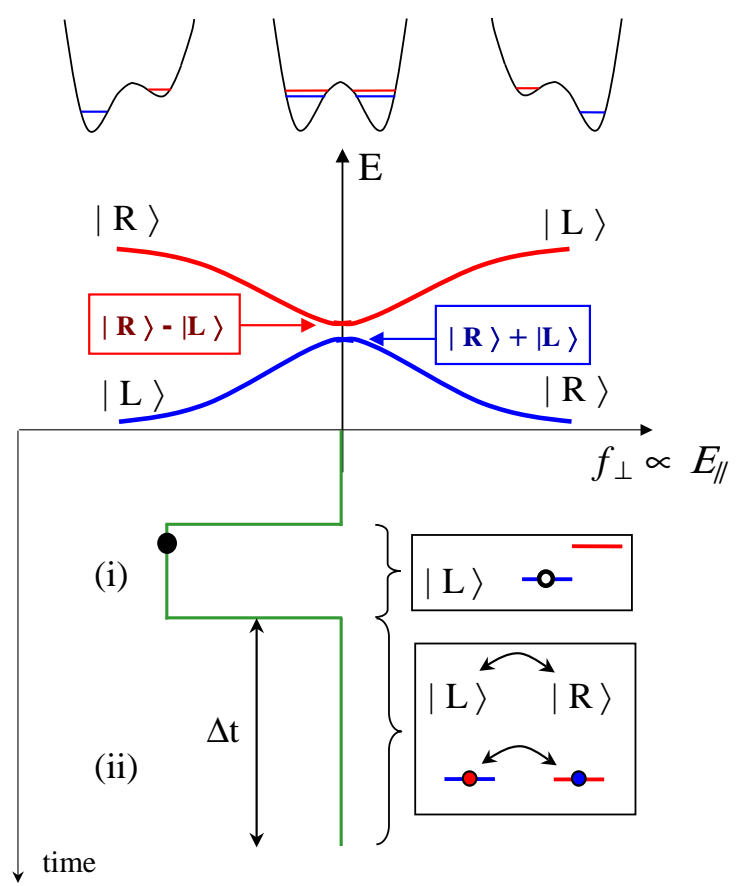

FIG. 3: A schematic diagram for controlling the quantum state of a nanobar via coherent oscillations at the degeneracy point. Tuning the system to its degeneracy point can be done by changing the perpendicular electric field $E_{\perp}$. For the case when $E_{\perp}$ and $E_{\|}$is zero at $t=0$, the perpendicular electrical field $E_{\perp}$ has to be applied first and then the longitudinal field $E_{\|}$(e.g., at the moment marked by the solid circle on the $E_{\perp}$ (time) curve). This initializes the system during the stage (i). Switching off the perpendicular electric field $E_{\perp}$ brings the system back to its degeneracy point, and the nanorod starts to oscillate a time $\Delta t$ during stage (ii). To observe coherent oscillations, measurements (e.g., optically or electrically) must be done for many values of $\Delta t$.

of nanobar qubits, one can again use the transverse electric field $E_{T}$ to tune selected qubits into resonance, then apply microwaves to perform conditional rotations and other operations.

For NEMS, and for more conventional applications such as resonators, major sources of noise ${ }^{32.33}$ (that limit the quality factor $Q$ ) are internal thermomechanical noise (such as heat flows due to inhomogeneous distribution of strain), Nyquist-Johnson noise from the driving electrical circuit, adsorption-desorption noise when a resonantor is moving in a non-vacuum, noise from moving defects, etc. In the quantum mechanical limit, some of these noises become unimportant. For example, there should be no heat flow: the nanobar should not be driven beyond its first excited state. The NEMS can be placed in a vacuum to reduce adsorption-desorption noise. The whole system, including its electrical components, has to be cooled in a dilution refrigerator in order to reach the two lowest

\begin{tabular}{|c|c|c|c|}
\hline System & SC charge QB & SC flux QB & Nano-bar QB \\
\hline & | excess charge > : & $\mid$ | current direction > : & | buckling direction > : \\
\hline States & $|0\rangle$ and $|1\rangle$ & $\mid\langle\rangle$ and $|P\rangle$ & $|R\rangle$ and $|L\rangle$ \\
\hline Hamiltonian & \multicolumn{3}{|c|}{$H=\epsilon \sigma_{z}+\hbar \Delta_{t} \sigma_{x}$} \\
\hline $\begin{array}{l}\text { Tunneling } \Delta_{t} \text { and } \\
\text { energy splitting } \\
\text { controlled by }\end{array}$ & $\begin{array}{l}\text { Gate voltage } V_{g} \\
\text { (normalized: } n_{g} \text { ) }\end{array}$ & $\begin{array}{l}\text { Magnetic flux: } \Phi \\
\text { (normalized: } \\
\quad f=\Phi / \Phi_{0} \text { ) }\end{array}$ & $\begin{array}{l}\text { Transverse force } f_{\perp} \\
\text { (induced, e.g., by a } \\
\text { transverse electric } \\
\text { field } E_{\perp} \text { ) }\end{array}$ \\
\hline $\begin{array}{l}\text { Tunneling } \\
\text { controlled by }\end{array}$ & $\begin{array}{l}\text { Josephson energy: } \\
\qquad \mathcal{E}_{J}(\Phi)\end{array}$ & $\begin{array}{c}\text { Josephson energy: } \\
\qquad \mathcal{E}_{J}\end{array}$ & $\begin{array}{l}\text { Either } \\
\text { - Longitudinal force } \\
\text { (induced by pressure or } \\
\text { electrical field } f_{\|} \text {) } \\
\text { - or its gradient } E_{\|} \\
\text {Longitudinal force and } \\
\text { its gradient control } \partial_{x} f_{\|} \\
\text {barrier height and its } \\
\text { curvature }\end{array}$ \\
\hline $\begin{array}{l}\text { Coupling } \\
\text { between qubits }\end{array}$ & $\begin{array}{l}\text { Electrical } \\
\text { (e.g., capacitive or } \\
\text { inductive coupling) }\end{array}$ & Magnetic & Electrical (dipolar) \\
\hline $\begin{array}{l}\text { Decoherence } \\
\text { sources include }\end{array}$ & Charge fluctuations & Flux fluctuations & $\begin{array}{l}\text { 1. Charge fluctuations } \\
\text { 2. Phonon-phonon } \\
\text { interactions }\end{array}$ \\
\hline Read-out & $\begin{array}{l}\text { Electrical } \\
\text { (e.g., SET or JJ) }\end{array}$ & Magnetic (SQUID) & $\begin{array}{l}\text { Either electric or } \\
\text { optical, or mechanical }\end{array}$ \\
\hline
\end{tabular}

Table: comparison of Josephson-junction superconducting (JJ SC) charge, JJ SC flux and nano-bar qubits (QB).

states of the nanobar, so that the Nyquist-Johnson noise is also suppressed. The main source of quantum mechanical decoherence might be internal dissipation caused by phonon-phonon interactions. Since the nanobars are clamped onto a much larger substrate, in which lower energy phonon modes exist, coupling at the base of the nanobars could lead to relaxation/excitation of the qubit states. However, the long-wave-length phonons produce a correlated noise, which can be reduced by working within a properly chosen decoherence-free subspace (see schematics in Fig. 2d and, e.g. $\left.{ }^{27.34}\right)$. Another source of decoherence would be charge fluctuations. Charged nanobars allow increased maneuverability of the NEMS; but the surrounding and internal charge fluctuators can produce charge noises that affect the relaxation and dephasing of the qubit.

Single-wall nanotubes are a natural candidate for the mechanical qubit we consider here. However, our proposal is not limited to them. Indeed, a multiple-wall nanotube could provide a higher-frequency mechanical oscillator and more favorable condition for observing macroscopic quantum tunnelling and the coherent evolution of mechanical motion. Silicon-based systems provide another enticing alternative, especially from the perspective of fabrication. While it might be difficult to clamp several single-wall nanotubes to make identical nanobars (with controlled inter-bar distance, same length, same buckling orientation, and same response to external stress), fabricating a lithographically-patterned silicon-based nanos- 
tructure would be much more reliable. $\mathrm{SiC}$ nanobars have shown higher stiffness ${ }^{22}$ compared to $\mathrm{Si}$. Unless nano-assembled nanotubes can be made with sufficiently high precision, materials that can be lithographically fabricated seem more promising candidates for a larger-scale mechanical quantum information processor. This would be an ironic turn of events, given that the first computers (by C. Babbage) were mechanical.

The detection of the nanobar state can be done either electrically $y^{6.18 .35}$ or optically ${ }^{36.37}$. It has been shown in recent experiments ${ }^{6}$ that single electron transistors are very sensitive to small charge displacements. Optical detectors can also be used, where light scattering can detect the state of the bent nanorod (Fig. 2e).

Very recently, Ref ${ }^{38}$ fabricated suspended nanobars (driven by a $25 \mathrm{MHz}$ current through an attached electrode) switching between two distinct states. These sus- pended nanobars have already been tested ${ }^{38}$ as very fast and very low-power-consumption storage memory devices. Still, many challenges lie ahead on the road to practical quantum electromechanics. We hope that our proposal here stimulate more research towards the ultimate quantum limit of NEMS.

\section{ACKNOWLEDGMENT}

This work was supported in part by the National Security Agency (NSA) and Advanced Research and Development Activity (ARDA) under Air Force Office of Research (AFOSR) contract number F49620-02-1-0334; and also supported by the US National Science Foundation grant No. EIA-0130383.
1 B. Reulet, A.Yu. Kasumov, M. Kociak, R. Deblock, I.I. Khodos, Yu.B. Gorbatov, V.T. Volkov, C. Journet, and H. Bouchiat, Phys. Rev. Lett. 85, 2829 (2000).

2 A. Izmalkov et al., Europhys. Lett. 65, 844 (2004).

3 A.N. Cleland, Foundations of Nanomechanics: From SolidState Theory to Device Applications (Springer, New York, 2003).

4 M. Roukes, Phys. World 14, 25 (2001); Sci. Am., 285, 48 (2001).

5 R.H. Blick, A Erbe, L. Pescini, A. Kraus, D.V. Scheible, F.W. Beil, E. Hoehberger, A. Hoerner, J. Kirschbaum, H. Lorenz and J.P. Kotthaus J. Phys.: Cond. Mat. 14, R905 (2002).

6 M.D. LaHaye, O. Buu, B. Camoratta, and K.C. Schwab, Science 304, 74-77 (2004).

7 K. Schwab, E.A. Henriksen, J.M. Worlock, and M.L. Roukes, Nature 404, 974-977 (2000).

8 M. Blencowe, Phys. Rep. 395, 159-222 (2004).

9 A. Gaidarzhy, G. Zolfagharkhani, R.L. Badzey, P. Mohanty, Phys. Rev. Lett. 94, 030402 (2005).

10 S.M. Carr, W.E. Lawrence, and M.N. Wybourne, Phys. Rev. B 64, 220101(R) (2001).

11 P. Werner, W. Zwerger, Europhys. Lett. 65, 158 (2004).

12 E. Buks, M.L. Roukes, JMEMS 11, 802-807 (2002).

13 J. Eisert et al., M.B. Plenio, S. Bose, J. Hartley, Phys. Rev. Lett. 93, 190402 (2004).

14 A.D. Armour, M.P. Blencowe, K.C. Schwab, Phys. Rev. Lett. 88, 148301 (2002).

15 A. Hopkins, K. Jacobs, S. Habib, K.C. Schwab, Phys. Rev. B 68, 235328 (2003); I. Wilson-Rae, P. Zoller, A. Imamoglu, Phys. Rev. Lett. 92, 075507 (2004); I. Martin, A. Shnirman, L. Tian, and P. Zoller, Phys. Rev. B 69, 125339 (2004).

16 L. Tian and P. Zoller, Phys. Rev. Lett. 93, 266403 (2004).

17 N. Nishiguchi, Phys. Rev. B, 68, 121305(R) (2003).

18 J. Kirschbaum, E.M. Höhberger, R.H. Blick, W. Wegscheider, M. Bichler, Appl. Phys. Lett. 81, 280 (2002).

19 I. Bargatin, M.L. Roukes, Phys. Rev. Lett. 91, 138302 (2003).
20 M.M.J. Treacy, T.W. Ebbesen, and J.M. Gibson, Nature 381, 678 (2002).

21 P. Poncharal, Z.L. Wang, D. Ugarte, and W.A. de Heer, Science 283, 1513-1516 (1999).

22 E.W. Wong, P.E. Sheehan, and C.M. Lieber, Science 277, 1971-1975 (1997).

23 Y. Nakamura, Yu.A. Pashkin, and J.S. Tsai, Nature 398, 786 (1999); Y.A Pashkin et al., Nature 421, 823 (2003); D. Vion et al., Science 296, 886 (2002); J.Q. You, F. Nori, Physics Today 58, No 11, p. 42(2005).

24 I. Chiorescu et al., Nature 431, 159 (2004).

25 J.M. Martinis et al., Phys. Rev. Lett. 89, 117901 (2002); R. McDermott et al., Science 307, 1299 (2005).

26 V. Ambegaokar, U. Eckern, G. Schön, Phys. Rev. Lett. 48, 17451748 (1982); U. Eckern, G. Schön, V. Ambegaokar, Phys. Rev. B 30, 6419 (1984).

27 S. Savel'evet al., unpublished.

28 L.D. Landau, E.M. Lifshitz, Mechanics (ButterworthHeinemann, Oxford, 1995).

29 S. Savel'ev, X. Hu, F. Nori, cond-mat/0412521 C.Q. Choi, Sci. Am. 29228 (2005); C.Q. Choi United Press International, April 8, 2005 (http://www.upi.com/inc/view.php?StoryID=20050317-124226-2271r

30 S. Savel'ev and F. Nori, Phys. Rev. B 70, 214415 (2004).

31 L.D. Landau, E.M. Lifshitz, Quantum Mechanics (Butterworth-Heinemann, Oxford, 1995).

32 A.N. Cleland and M.L. Roukes, J. Appl. Phys. 92, 2758 (1998).

33 A.S. Nowick and B.S. Berry, Inelastic Relaxation in Crystalline Solids (Academic Press, New York, 1972).

34 P. Zanardi, M. Rasetti, Phys. Rev. Lett. 79, 3306 (1997); J.Q. You, X. Hu, F. Nori, cond-mat/0407423

35 R.G. Knobel, A.N. Cleland, Nature 424, 291-293 (2003).

36 H.J. Mamin et al., Phys. Rev. Lett. 91, 207604 (2003).

37 I. Kiyat, C. Kocabas, A. Aydinli, J. Micromech. Microeng. 14, 374 (2004).

38 R.L. Badzey et al., Appl. Phys. Lett. 85, 3587 (2004). 